\title{
COMPOSIÇÃO FLORÍSTICA E ESTRUTURA DO ESTRATO INFERIOR DA FLORESTA DE VÁRZEA NA ÁREA DE PROTEÇÃO AMBIENTAL ILHA DO COMBU, MUNICÍPIO DE BELÉM, ESTADO DO PARÁ ${ }^{1}$
}

\author{
Bernardo Antônio Rodrigues Maués², Mário Augusto Gonçalves Jardim², Fábio de Jesus Batista³, \\ Tonny David Santiago Medeiros² e Adriano da Costa Quaresma²
}

\begin{abstract}
RESUMO - O estrato inferior é formado pela regeneração das espécies arbóreas, arbustivas, herbáceas, epífitas e lianas formando um nicho ecológico de vital importância para o estabelecimento e desenvolvimento da floresta. Com o objetivo de analisar a composição florística e a estrutura do estrato inferior da floresta de várzea na APA Ilha do Combu, Belém, Pará, foram alocadas 50 parcelas de 50 x 4 m e divididas em 25 subparcelas de 2 x $2 \mathrm{~m}$. Foram identificadas e quantificadas todas as espécies com Diâmetro à Altura do Peito (DAP) d” $10 \mathrm{~cm}$. Calcularam-se a diversidade, densidade e frequência relativas, categoria de tamanho relativa e regeneração natural relativa. Foram amostrados 22.221 indivíduos, 67 famílias, 153 gêneros e 223 espécies, e o índice de Shannon (H') foi de 3,72 nat/ind e a equabilidade (J'), de 0,69. Fabaceae, Malvaceae e Arecaceae destacaram-se em riqueza de espécies e Euterpe oleracea e Virola surinamensis em densidade relativa, categoria de tamanho relativa e regeneração natural relativa. O hábito arbóreo apresentou o maior número de espécies e indivíduos nas classes de tamanhos 1 e 2. Os mecanismos de adaptação e a produção de frutos estão relacionados com a diversidade da área, onde as espécies com estratégias mais eficientes são dominantes e mais representativas quantitativamente na comunidade.
\end{abstract}

Palavras-chave: Regeneração, Riqueza e classes de tamanho e Unidade de conservação.

\section{FLORISTIC COMPOSITION AND STRUCTURE OF LOWER STRATUM OF FLOODPLAIN FOREST IN ILHA DO COMBU ENVIRONMENTAL PROTECTION AREA, BELÉM, STATE OF PARÁ}

\begin{abstract}
The lower stratum is formed by the regeneration of arboreous, arbustive, herbaceous, epiphyte, and liana species, forming an ecological niche of vital importance to the establishment and development of the forest. To analyze floristic composition and structure of the lower stratum of the floodplain forest, it was allocated fifty $50 \times 4-m$ plots, divided in $252 \times 2-m$ subplots. All species with diameter at breast height $(\mathrm{DBH}) \leq 10 \mathrm{~cm}$ were identified and quantified. Relative diversity, density and frequency, relative category of size, and relative natural regeneration were calculated. It was sampled 22,221 individuals, 67 families, 153 genera and 223 species, and the Shannon index ( $\left.H^{\prime}\right)$ was 3.72 nat/ind and Equability ( $J$ ') was 0.69. Fabaceae, Malvaceae and Arecaceae stood out for species richness, and Euterpe oleracea and Virola surinamensis for relative density, relative category of size, and relative natural regeneration. The arboreous habit presented the greatest number of species and individuals in the size classes 1 and 2. The adaptation mechanisms and fruit production are related to the diversity of the area, where the species with the most efficient strategies are dominant and more quantitatively representatives in the community.
\end{abstract}

Keywords:Regeneration, Richness and size classes and Conservation unity.

\footnotetext{
${ }^{1}$ Recebido em 02.12.2008 e aceito para publicação em 18.04.2011.

${ }^{2}$ Museu Paraense Emilio Goeldi, MPEG, Brasil. E-mail: <bmfloresta@yahoo.com.br>, <jardim@museu_goeldi.br>, $<$ tjahbio@yahoo.com.br>e <tatoquaresma@hotmail.com>.

${ }^{3}$ Universidade Federal Rural da Amazônia, UFRA, Brasil. E-mail: <fabiojb@superig.com.br>.
} 


\section{INTRODUÇÃO}

A floresta de várzea corresponde a segunda maior formação vegetal da região Amazônica, ocupando $75.880,8$ km², cerca de 1,6\% do bioma amazônico com áreas inundáveis formadas por solos aluviais resultantes de contínua sedimentação de partículas suspensas nas águas dos rios (SANTOS et al., 2004). A vegetação desenvolve-se sob inundações diárias causadas pelo fluxo das marés com frequentes adaptações ecológicas, fisiológicas e morfológicas (ALMEIDA et al., 2004), podendo influenciar na dominância de determinadas espécies da regeneração (RABELO et al., 2000).

Para Gama et al. (2003), a floresta de várzea começou a ser explorada no início da década de 1950, quando os primeiros trabalhos de inventário florestal já acenavam para a inclusão da análise da estrutura da regeneração natural no entendimento da dinâmica da floresta. Algumas espécies apresentam potencial econômico, como as plantas oleaginosas (Mauritia flexuosa Mart. e Carapa guianensis Aubl.), frutíferas (Euterpe oleracea Mart. e Spondias mombin L.), lactíferas (Hevea brasiliensis (Willd. ex A.Juss.) Muell. Arg. e Symphonia globulifera L.) e madeireiras (Swartzia racemosa Benth. e Virola surinamensis (Rol. ex Rottb.) Warb), consideradas como fonte de renda para os povos ribeirinhos (JARDIM; VIEIRA, 2001; RODRIGUES et al., 2006), todavia estão sendo dizimadas pela exploração desordenada e pela ausência de manejo (CARIM et al., 2008).

No Brasil, especificamente na Amazônia, é incipiente o conhecimento sobre as espécies que constituem o estrato inferior das florestas de várzea em unidades de conservação. Em UCs do Pará, os estudos mais recentes foram realizados por Batista (2008), em dois fragmentos florestais na Reserva Extrativista Chocoaré, Mato Grosso, no Município de Santarém Novo, e por Jardim (2000) na Área de Proteção Ambiental, Ilha do Combu, no Município de Belém (PA). No entanto, quando se considera a extensão territorial ocupada pela floresta de várzea, os estudos ainda são insuficientes dos pontos de vista quantitativo e qualitativo.

Consequentemente, para entender a dinâmica de uma floresta não basta apenas o entendimento da regeneração das espécies arbóreas, é preciso conhecer a composição e estrutura do estrato inferior, incluindo as espécies herbáceas, arbustivas, lianas e epífitas. De acordo com Oliveira e Amaral (2005), essas espécies, juntamente com a regeneração das espécies arbóreas, formam um nicho ecológico importante para o estabelecimento e desenvolvimento das populações que constituirão o estrato superior. Considerando a relevância do assunto, o objetivo deste estudo foi analisar a composição florística e a estrutura do estrato inferior da floresta de várzea localizada na Área de Proteção Ambiental Ilha do Combu, Município de Belém, Pará.

\section{MATERIAL E MÉTODOS}

O estudo foi realizado na Área de Proteção Ambiental Ilha do Combu, Município de Belém, PA, que abrange uma área de aproximadamente $15 \mathrm{~km}^{2}$ (48 $25^{\circ}$ ' W $1^{\circ} 25^{\prime} \mathrm{S}$ ), ao sul da cidade de Belém (1,5 km por via fluvial), margem esquerda do rio Guamá. O clima é do tipo Am, segundo a classificação de Köppen, com precipitação média anual de $2.500 \mathrm{~mm}$ e temperatura média anual de $27^{\circ} \mathrm{C}$ (JARDIM, 2000). O solo é do tipo Glei Pouco Húmico, com alta percentagem de siltes, argila e baixa percentagem de areia (SILVA; SAMPAIO, 1998). A ilha abrange uma floresta de várzea composta por árvores, arbustos, herbáceas, lianas e espécies de sub-bosque (RODRIGUES et al., 2006).

A coleta de dados foi realizada de novembro de 2007 a abril de 2008. Foram alocadas, sistematicamente em vários pontos da floresta, 50 parcelas de 50 × 4 m, divididas em 25 subparcelas de 2 × $2 \mathrm{~m}\left(4 \mathrm{~m}^{2}\right)$, dispostas alternadamente. Foram identificadas e quantificadas todas as espécies com diâmetro à altura do peito (DAP) $\leq 10 \mathrm{~cm}$. A identificação foi realizada por Carlos Alberto da Silva, técnico botânico do Museu Paraense Emílio Goeldi. Os sistemas de classificações utilizados nas nomenclaturas botânicas foram APG II (2003) para angiospermas, Smith et al. (2006) para as monilophytas e Kramer e Green (1990) para as licophytas.

Para a quantificação das espécies foram consideradas três classes de tamanho, conforme a metodologia recomendada por Finol (1971): Ct1: < 0,3 m de altura; Ct2: $\geq 0,3 \mathrm{~m} \mathrm{e}<1,0 \mathrm{~m}$; Ct3: e < 1,0 m e DAP $\leq 10 \mathrm{~cm}$ e a inclusão dos indivíduos nas classes com o auxílio de uma vara ( $1 \mathrm{~m}$ de altura) posicionada no centro das subparcelas.

A composição florística foi avaliada através da distribuição dos indivíduos em famílias, gêneros e espécies. Por meio do índice de Shannon-Wiener (H’) (MAGURRAN, 1988) e da equabilidade (E`) (PIELOU, 1977), foi avaliada a diversidade de espécies. A caracterização das espécies quanto ao hábito foi baseada

Revista Árvore, Viçosa-MG, v.35, n.3, Edição Especial, p.669-677, 2011 
em Ribeiro et al. (1999) e consultas às exsicatas do Herbário MG. Os parâmetros fitossociológicos calculados foram a densidade e frequência absolutas e relativas, a categoria de tamanho relativa por espécie (Ct\%) conforme Finol (1971) e Lima Filho et al. (2002) e a regeneração natural relativa (Rn\%) (Finol, 1971):

$$
\begin{gathered}
\mathrm{Ct} \%=\frac{\text { Ct.abs x } 100}{\text { ÓCt.abs }} \\
\text { Ct.abs }=\text { VF }(\mathrm{Cti}) \times \mathrm{n}(\mathrm{Cti})+\mathrm{VF}(\mathrm{Ctii}) \times \mathrm{n}(\mathrm{Ctii}) \\
+\mathrm{VF}(\text { Ctiii }) \times \mathrm{n}(\mathrm{Ctiii}) \\
\mathrm{Rn} \%=\frac{\mathrm{Ab} \%+\text { Freq } \%+\mathrm{ct} \%}{3}
\end{gathered}
$$

em que:

$\mathrm{Ct} \%=$ categoria de tamanho relativa;

Ct.abs = categoria de tamanho absoluta;

$\mathrm{VF}=$ valor fitossociológico simplificado (dividido por 10) correspondente à proporção de cada indivíduo em cada categoria de tamanho;

Cti $=$ categoria de tamanho 1 ;

Ctii $=$ categoria de tamanho 2 ;

Ctiii $=$ categoria de tamanho 3 ;

$\mathrm{Ab} \%$ = abundância relativa; $\mathrm{e}$

Freq\% = frequência relativa.

\section{RESULTADOS}

Foram registrados 64 famílias, 153 gêneros, 223 espécies e 22.221 indivíduos. As famílias Fabaceae (36 espécies), Malvaceae (12) e Arecaceae (11) foram as mais representativas em espécies. Os gêneros que se destacaram com maior número de indivíduos foram Inga (3253), Euterpe (2597) e Virola (1932). Euterpe oleracea (11,69\% do número total de indivíduos) e Virola surinamensis (8,69\%) apresentaram maior densidade relativa. Essas espécies, juntamente com Inga Alba, Memora magnifica, Pterocarpus officinalis, Piper hispidum, Hevea brasiliensis, Symphonia globulifera, Inga edulis, Ischnosiphon obliquus e Hymenocallis sp., foram as mais frequentes. E.oleracea e V.surinamensis também apresentaram os maiores valores de Ct\% e Rn\% (Tabela 1). Os valores do índice de diversidade de espécies de Shannon-Wiener $\left(\mathrm{H}^{`}\right)$ e a equabilidade ( $E^{`}$ ) foram 3,72 e 0,69 , respectivamente.
O hábito arbóreo concentrou o maior número de espécies (110), seguido por liana (45), herbáceo (34), arbustivo (18), palmeira (9), hemiepífita (5) e epífita (2) e representou 54,71\% (12.157) indivíduos, distribuídos nas classes de tamanhos 1 (7989), 2 (3399) e 3 (769), seguidos por palmeiras (3556) (Figura 1). Hemiepífita e epífita com 165 e 7 indivíduos, respectivamente, foram menos representativas. O maior número de invidíduos (12911) esteve presente na Ct1 e mais representados por V. surinamensis (1558), I. alba (1506) e E. oleracea (1385) na Ct2 (6.843 indivíduos), sendo estes representados por $S$. globulifera (728) e E. oleracea (585), e na Ct3 (2467), por E. oleracea (627) e I. obliquus (311) (Figura 2A-C).

\section{DISCUSSÃO}

Fabaceae, Malvaceae e Arecaceae estão como as mais ricas em espécies em florestas de várzea (RABELO et al., 2000; GAMA et al., 2002; BATISTA, 2008), pelo fato de algumas espécies apresentarem mecanismos adaptativos na estrutura radicular para fixação de nitrogênio em áreas inundáveis (JARDIM; VIEIRA, 2001; ALMEIDA et al., 2004; CARIM et al., 2008), que influenciam no crescimento e no desenvolvimento (ATWELL; STEER, 1990; JACKSON; DREW, 1984; KOZLOWSKI; PALLARDY, 1984), consistindo na preponderância numérica de espécies de uma mesma família, característica comum em florestas tropicais (RICHARDS, 1981). Para Brito et al. (2008), as inundações sazonais do solo influenciam na germinação, estabelecimento e dominância das espécies arbóreas; além da posição topográfica e da umidade na formação de grupos florísticos (JARDIM; VIEIRA, 2001; NARVAES et al., 2008).

O índice de diversidade de Shannon $\left(\mathrm{H}^{\star}=3,72\right.$ nat.ind $\left.^{-1}\right)$ e a equabilidade $\left(E^{\varsigma}=0,69\right)$ foram similares aos registrados no componente arbustivo-arbóreo por Bianchini et al. (2003) e Kurtz et al. (2009) e acima dos valores registrados por Batista (2008), Gama et al. (2002) e Budke et al. (2004), no componente arbóreo. Os valores similares indicam que algumas espécies estão com altas densidades e outras com baixo número de indivíduos, e os valores acima desses são devidos à inclusão de outros hábitos além do arbóreo.

O hábito arbóreo com maior número espécies e indivíduos nas primeiras classes de tamanho também foi constatado nos estudos de Jardim (2000), Gama et al. (2001), Jardim e Vieira (2001), Bentes-Gama et 
Tabela 1 - Parâmetros fitossociológicos das espécies do estrato inferior amostradas em 0,5 ha de floresta de várzea na APA Ilha do Combu, Município de Belém, Pará. Hábito = (Arv: arbóreo, Arb: arbustivo, Ep: epífita, Hem: hemiepífita, Herb: herbáceo, Lia: liana e Pal: palmeira), Dr\% = Densidade relativa, Fr\% = Frequência relativa, Ct\% = Categoria de tamanho relativo e Rn\% = Regeneração natural relativa.

Table 1 - Fitossociology parameters of species in the lower stratum in 0.5 ha of APA Ilha do Combú floodplain forest, municipality of Belém, Pará. Habit = (Arv: arboreous, Arb: arbustive, Ep: epiphyte, Hem: hemiepiphyte, Herb: herbaceous, Lia: liana and Pal: palm tree); Dr\% = Relative density; Fr\% = Relative frequency; Ct\% = Category of relative size and $R n \%=$ Natural relative regeneration.

\begin{tabular}{|c|c|c|c|c|c|c|}
\hline Família & Espécie & Hábito & Dr \% & Fr \% & $\mathrm{Ct} \%$ & $\operatorname{Rn}(\%)$ \\
\hline$\overline{\text { Arecaceae }}$ & Euterpe oleracea Mart. & Pal & 11,69 & 2,55 & 10,67 & 8,30 \\
\hline Myristicaceae & Virola surinamensis (Rol. ex Rottb.) Warb. & Arv & 8,69 & 2,55 & 10,24 & 7,16 \\
\hline Fabaceae & Inga alba (Sw.) Willd. & Arv & 7,37 & 2,40 & 9,21 & 6,33 \\
\hline Fabaceae & Inga edulis Mart. & Arv & 6,25 & 2,15 & 7,66 & 5,35 \\
\hline Clusiaceae & Symphonia globulifera L. f. & Arv & 6,26 & 2,20 & 5,73 & 4,73 \\
\hline Fabaceae & Pterocarpus officinalis Jacq. & Arv & 4,44 & 2,40 & 4,79 & 3,88 \\
\hline Marantaceae & Ischnosiphon obliquus (Rudge) Körn. & Herb & 4,39 & 2,10 & 3,15 & 3,21 \\
\hline Araceae & Dieffenbachia humilis Poepp. & Herb & 3,09 & 2,04 & 3,29 & 2,81 \\
\hline Piperaceae & Piper hispidum Sw. & Arb & 2,92 & 2,30 & 2,99 & 2,74 \\
\hline Fabaceae & Macrolobium bifolium (Aubl.) Pers. & Arv & 2,74 & 1,64 & 2,97 & 2,45 \\
\hline Euphorbiaceae & Hevea brasiliensis (Willd. ex A. Juss.) Müll. Arg. & Arv & 2,53 & 2,25 & 2,00 & 2,26 \\
\hline Bignoniaceae & Memora magnifica (Mart. ex DC.) Bureau & Lia & 1,72 & 2,45 & 1,46 & 1,88 \\
\hline Acanthaceae & Trichanthera gigantea (Bonpl.) Nees & Arv & 2,09 & 1,18 & 2,19 & 1,82 \\
\hline Euphorbiaceae & Hura crepitans L. & Arv & 1,74 & 1,99 & 1,54 & 1,76 \\
\hline Piperaceae & Piper sp. 1 & Arb & 1,54 & 1,74 & 1,45 & 1,58 \\
\hline Amaryllidaceae & Hymenocallis sp. & Herb & 1,20 & 2,10 & 1,34 & 1,54 \\
\hline Heliconiaceae & Heliconia bihai (L.) L. & Herb & 1,64 & 1,69 & 1,28 & 1,53 \\
\hline Fabaceae & Zygia latifolia (L.) Fawc. \& Rendle & Arb & 1,43 & 1,79 & 1,16 & 1,46 \\
\hline Araceae & Montrichardia arborescens (L.) Schott & Herb & 1,67 & 1,69 & 0,87 & 1,41 \\
\hline Malvaceae & Byttneria coriacea Britton & Lia & 1,30 & 1,58 & 1,26 & 1,38 \\
\hline Arecaceae & Astrocaryum murumuru Mart. & Pal & 1,41 & 1,12 & 1,56 & 1,37 \\
\hline Piperaceae & Piper arboreum Aubl. & Arb & 1,26 & 1,28 & 1,12 & 1,22 \\
\hline Meliaceae & Trichilia singularis C. DC. & Arv & 0,85 & 1,58 & 1,04 & 1,16 \\
\hline Malvaceae & Theobroma сасао L. & Arv & 1,04 & 1,48 & 0,95 & 1,16 \\
\hline Meliaceae & Carapa guianensis Aubl. & Arv & 0,77 & 1,79 & 0,52 & 1,03 \\
\hline Bignoniaceae & Memora flavida (DC.) Bureau \& K. Schum. & Lia & 0,70 & 1,58 & 0,72 & 1,00 \\
\hline \multirow[t]{2}{*}{ Rubiaceae } & Psychotria colorata (Willd. & & & & & \\
\hline & ex Roem. \& Schult.) Müll. Arg. & Arb & 0,77 & 1,12 & 0,96 & 0,95 \\
\hline Dioscoreaceae & Dioscorea laxiflora Mart. ex Griseb. & Lia & 0,52 & 1,79 & 0,44 & 0,92 \\
\hline Chrysobalanaceae & Licania guianensis (Aubl.) Griseb. & Arv & 0,71 & 1,38 & 0,56 & 0,88 \\
\hline Burseraceae & Protium krukoffii Swart. & Arv & 0,46 & 1,18 & 0,45 & 0,69 \\
\hline Fabaceae & Cynometra marginata Benth. & Arv & 0,81 & 0,26 & 0,98 & 0,68 \\
\hline Fabaceae & Machaerium leiophyllum (DC.) Benth. & Lia & 0,48 & 1,02 & 0,51 & 0,67 \\
\hline Meliaceae & Guarea kunthiana A. Juss. & Arv & 0,63 & 0,61 & 0,75 & 0,66 \\
\hline Fabaceae & Pentaclethra macroloba (Willd.) Kuntze & Arv & 0,44 & 1,18 & 0,37 & 0,66 \\
\hline Euphorbiaceae & Manihot tripartita (Spreng.) Mull. Arg. & Herb & 0,61 & 0,61 & 0,68 & 0,63 \\
\hline Anacardiaceae & Spondias mombin L. & Arv & 0,40 & 1,02 & 0,36 & 0,59 \\
\hline Malvaceae & Matisia paraensis Huber & Arv & 0,40 & 0,97 & 0,35 & 0,57 \\
\hline Fabaceae & Inga cordatoalata Ducke & Arv & 0,36 & 0,77 & 0,39 & 0,51 \\
\hline Dryopteridaceae & Polybotrya caudata Kunze & Hem & 0,42 & 0,61 & 0,39 & 0,47 \\
\hline Malvaceae & Quararibea guianensis Aubl. & Arv & 0,32 & 0,87 & 0,23 & 0,47 \\
\hline Rubiaceae & Genipa americana L. & Arv & 0,20 & 1,02 & 0,16 & 0,46 \\
\hline Fabaceae & Machaerium ferox (Mart. ex Benth.) Ducke & Lia & 0,31 & 0,66 & 0,40 & 0,46 \\
\hline Acanthaceae & Justicia pseudoamazonica Lindau & Herb & 0,40 & 0,41 & 0,51 & 0,44 \\
\hline Chrysobalanaceae & Parinari excelsa Sabine & Arv & 0,30 & 0,66 & 0,34 & 0,44 \\
\hline Araceae & Philodendron sp. 2 & Hem & 0,29 & 0,72 & 0,29 & 0,43 \\
\hline
\end{tabular}

Revista Árvore, Viçosa-MG, v.35, n.3, Edição Especial, p.669-677, 2011 
Tabela 1 - Cont.

Table 1 - Cont.

\begin{tabular}{|c|c|c|c|c|c|c|}
\hline Pteridaceae & Adiantum tomentosum Klotzsch & Herb & 0,20 & 0,82 & 0,23 & 0,42 \\
\hline Malvaceae & Herrania mariae (Mart.) Decne. ex Goudot & Arv & 0,18 & 0,87 & 0,16 & 0,40 \\
\hline Lecythidaceae & Gustavia augusta L. & Arv & 0,23 & 0,82 & 0,16 & 0,40 \\
\hline Fabaceae & Crudia bracteata Benth. & Arv & 0,31 & 0,56 & 0,31 & 0,39 \\
\hline Thelypteridaceae & Thelypteris hispidula (Decne.) C.F. Reed. & Lia & 0,32 & 0,51 & 0,31 & 0,38 \\
\hline Bignoniaceae & Arrabidaea sp. & Lia & 0,31 & 0,51 & 0,30 & 0,38 \\
\hline Sapindaceae & Serjania paucidentata DC. & Lia & 0,16 & 0,82 & 0,12 & 0,37 \\
\hline Sapotaceae & Sarcaulus brasiliensis (A. DC.) Eyma & Arv & 0,19 & 0,72 & 0,17 & 0,36 \\
\hline Fabaceae & Inga brachyrhachis Harms & Arv & 0,45 & 0,05 & 0,56 & 0,35 \\
\hline Moraceae & Ficus maxima Mill. & Arv & 0,18 & 0,66 & 0,19 & 0,35 \\
\hline Clusiaceae & Rheedia macrophylla (Mart.) Planch. \& Triana & Arv & 0,17 & 0,72 & 0,14 & 0,34 \\
\hline Arecaceae & Socratea exorrhiza (Mart.) H. Wendl. & Pal & 0,30 & 0.46 & 0.26 & 0.34 \\
\hline Hippocrateaceae & Prionostemma asperum (Lam.) Miers & Lia & 0,25 & 0,56 & 0,19 & 0,33 \\
\hline Bignoniaceae & Cydista aequinoctialis (L.) Miers & Lia & 0,13 & 0,77 & 0,09 & 0,33 \\
\hline Malvaceae & Pavonia fruticosa (Mill.) Fawc. \& Rendle & Herb & 0,18 & 0,61 & 0,18 & 0,33 \\
\hline Arecaceae & Desmoncus polyacanthos Mart. & Lia & 0,16 & 0,72 & 0,09 & 0,32 \\
\hline Heliconiaceae & Heliconia acuminata Rich. & Herb & 0,12 & 0,61 & 0,14 & 0,29 \\
\hline Araceae & Urospatha sp. & Herb & 0,18 & 0,56 & 0,13 & 0,29 \\
\hline Hippocrateaceae & Hippocratea sp. & Lia & 0,23 & 0,36 & 0,28 & 0,29 \\
\hline Arecaceae & Geonoma macrostachys Mart. & Pal & 0,09 & 0,61 & 0,08 & 0,26 \\
\hline Lomariopsidaceae & Lomariopsis japurensis (Mart.) J. Sm. & Herb & 0,12 & 0,51 & 0,13 & 0,25 \\
\hline Fabaceae & Pterocarpus amazonicus Huber & Arv & 0,10 & 0,56 & 0,09 & 0,25 \\
\hline Fabaceae & Swartzia racemosa Benth. & Arv & 0,17 & 0,41 & 0,17 & 0,25 \\
\hline Malpighiaceae & Hiraea chrysophylla A. Juss. & Lia & 0,18 & 0,36 & 0,20 & 0,24 \\
\hline Fabaceae & Crudia glaberrima (Steud.) J.F. Macbr. & Arv & 0,14 & 0,46 & 0,14 & 0,24 \\
\hline Meliaceae & Trichilia quadrijuga Kunth & Arv & 0,26 & 0,15 & 0,31 & 0,24 \\
\hline Violaceae & Rinorea passoura Kuntze & Arv & 0,18 & 0,41 & 0,12 & 0,24 \\
\hline Lomariopsidaceae & Lomariopsis prieuriana Fee & Herb & 0,14 & 0,41 & 0,12 & 0,22 \\
\hline Piperaceae & Piper sp. 2 & Arb & 0,22 & 0,31 & 0,14 & 0,22 \\
\hline Fabaceae & Inga nobilis Willd. & Arv & 0,11 & 0,46 & 0,09 & 0,22 \\
\hline Dilleniaceae & Davilla rugosa Poir. & Lia & 0,11 & 0,36 & 0,14 & 0,20 \\
\hline Combretaceae & Terminalia dichotoma G. Mey. & Arv & 0,15 & 0,31 & 0,14 & 0,20 \\
\hline Bignoniaceae & Macfadyena uncata (Andrews) Sprague \& Sandwith & Lia & 0,12 & 0,36 & 0,10 & 0,19 \\
\hline Marantaceae & Calathea sp. 2 & Herb & 0,14 & 0,26 & 0,17 & 0,19 \\
\hline Sapotaceae & Pouteria macrocarpa (Mart.) D. Dietr. & Arv & 0,13 & 0,31 & 0,12 & 0,19 \\
\hline Myrtaceae & Eugenia muricata DC. & Arv & 0,05 & 0,46 & 0,03 & 0,18 \\
\hline Thelypteridaceae & Thelypteris interrupta (Willd.) K. Iwats. & Lia & 0,12 & 0,31 & 0,11 & 0,18 \\
\hline Fabaceae & Dalbergia monetaria L. f. & Lia & 0,09 & 0,36 & 0,06 & 0,17 \\
\hline Moraceae & Ficus insipida Willd. & Arv & 0,06 & 0,41 & 0,04 & 0,17 \\
\hline Melastomataceae & Clidemia hirta (L.) D. Don & Arb & 0,08 & 0,36 & 0,08 & 0,17 \\
\hline Arecaceae & Bactris major Jacq. & Pal & 0,15 & 0,26 & 0,07 & 0,16 \\
\hline Urticaceae & Cecropia palmata Willd. & Arv & 0,08 & 0,36 & 0,04 & 0,16 \\
\hline Monimiaceae & Siparuna guianensis Aubl. & Arv & 0,15 & 0,15 & 0,16 & 0,15 \\
\hline Fabaceae & Mora paraensis (Ducke) Ducke & Arv & 0,09 & 0,31 & 0,07 & 0,15 \\
\hline Hernandiaceae & Hernandia guianensis Aubl. & Arv & 0,11 & 0,26 & 0,09 & 0,15 \\
\hline Sapotaceae & Pouteria sp. & Arv & 0,14 & 0,10 & 0,17 & 0,14 \\
\hline Fabaceae & Pterocarpus santalinoides L’Hér. ex DC. & Arv & 0,05 & 0,31 & 0,05 & 0,14 \\
\hline Solanaceae & Solanum sp. & Arb & 0,07 & 0,26 & 0,07 & 0,13 \\
\hline Myrtaceae & Eugenia tapacumensis $\mathrm{O}$. Berg & Arv & 0,14 & 0,05 & 0,19 & 0,13 \\
\hline Combretaceae & Combretum laxum Jacq. & Lia & 0,07 & 0,20 & 0,08 & 0,12 \\
\hline Passifloraceae & Passiflora vespertilio L. & Lia & 0,05 & 0,26 & 0,05 & 0,12 \\
\hline Piperaceae & Piper asperum Pers. & Arb & 0,13 & 0,10 & 0,11 & 0,11 \\
\hline Fabaceae & Crudia oblonga Benth. & Arv & 0,08 & 0,20 & 0,06 & 0,11 \\
\hline Melastomataceae & Miconia ciliata (Rich.) DC. & Arb & 0,03 & 0,26 & 0,02 & 0,10 \\
\hline
\end{tabular}


Tabela 1 - Cont.

Table 1 - Cont.

\begin{tabular}{|c|c|c|c|c|c|c|}
\hline Cyperaceae & Scleria pterota C. Presl. & Herb & 0,05 & 0,20 & 0,04 & 0,10 \\
\hline Arecaceae & Desmoncus mitis Mart. & Lia & 0,02 & 0,26 & 0,01 & 0,10 \\
\hline Pteridaceae & Adiantum latifolium Lam. & Herb & 0,09 & 0,10 & 0,10 & 0,10 \\
\hline Passifloraceae & Passiflora foetida $\mathrm{L}$. & Lia & 0,04 & 0,20 & 0,04 & 0,10 \\
\hline Connaraceae & Connarus perrottetii (DC.) Planch. & Arv & 0,08 & 0,15 & 0,06 & 0,10 \\
\hline Malvaceae & Sterculia pruriens (Aubl.) K. Schum. & Arv & 0,05 & 0,15 & 0,06 & 0,09 \\
\hline Bignoniaceae & Memora sp. 1 & Lia & 0,05 & 0,15 & 0,06 & 0,09 \\
\hline Melastomataceae & Mouriri guianensis Aubl. & Arv & 0,05 & 0,15 & 0,05 & 0,09 \\
\hline Malvaceae & Bombax munguba Mart. \& Zucc. & Arv & 0,03 & 0,20 & 0,02 & 0,08 \\
\hline Piperaceae & Piper aduncum L. & Arb & 0,08 & 0,10 & 0,06 & 0,08 \\
\hline Acanthaceae & Justicia dubiosa Lindau & Herb & 0,04 & 0,15 & 0,05 & 0,08 \\
\hline Malvaceae & Sterculia speciosa K. Schum. & Arv & 0,02 & 0,20 & 0,01 & 0,08 \\
\hline Dennstaedtiaceae & Pteridium sp. & Herb & 0,04 & 0,15 & 0,05 & 0,08 \\
\hline Orchidaceae & Vanilla mexicana Mill. & Lia & 0,02 & 0,20 & 0,01 & 0,08 \\
\hline Fabaceae & Bauhinia guianensis Aubl. & Arb & 0,04 & 0,15 & 0,03 & 0,07 \\
\hline Cucurbitaceae & Gurania sp. & Lia & 0,03 & 0,15 & 0,04 & 0,07 \\
\hline Piperaceae & Piper sp. 3 & Arb & 0,04 & 0,15 & 0,03 & 0,07 \\
\hline Araceae & Montrichardia sp. & Herb & 0,03 & 0,15 & 0,03 & 0,07 \\
\hline Dilleniaceae & Doliocarpus dentatus (Aubl.) Standl. & Lia & 0,03 & 0.15 & 0.03 & 0.07 \\
\hline Fabaceae & Inga sp. 1 & Arv & 0.03 & 0,15 & 0,03 & 0,07 \\
\hline Fabaceae & Inga sp. 2 & Arv & 0,03 & 0,15 & 0,02 & 0,07 \\
\hline Fabaceae & Swartzia polyphylla DC. & Arv & 0,03 & 0,15 & 0,02 & 0,07 \\
\hline Lecythidaceae & Heisteria sp. & Arv & 0,02 & 0,15 & 0,02 & 0,07 \\
\hline Arecaceae & Geonoma baculifera (Poit.) Kunth & Pal & 0,02 & 0,15 & 0,02 & 0,06 \\
\hline Hippocrateaceae & Pristimera nervosa (Miers) A. C. Sm. & Lia & 0,08 & 0,05 & 0,06 & 0,06 \\
\hline Chrysobalanaceae & Licania heteromorpha Benth. & Arv & 0,02 & 0,15 & 0,01 & 0,06 \\
\hline Lauraceae & Mezilaurus mahuba (A. Samp.) van der Werff & Arv & 0,02 & 0,15 & 0,01 & 0,06 \\
\hline Marantaceae & Calathea sp.1 & Herb & 0,07 & 0,05 & 0,06 & 0,06 \\
\hline Fabaceae & Inga laurina (Sw.) Willd. & Arv & 0,02 & 0,15 & 0,01 & 0,06 \\
\hline Araceae & Caladium bicolor (Aiton) Vent. & Herb & 0,05 & 0,05 & 0,07 & 0,06 \\
\hline
\end{tabular}

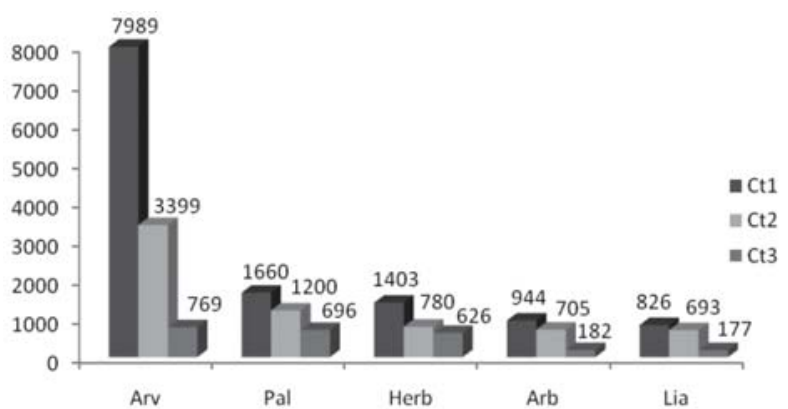

Figura 1-Número de indivíduos por hábito e classes de tamanho do estrato inferior em 0,5 ha de floresta de várzea na APA Ilha do Combu, Município de Belém, Pará. Arv: arbóreo, Pal:palmeira, Herb: herbáceo, Arb: arbustivo e Lia: liana. Ct1: classe de tamanho 1, Ct2: classe de tamanho 2 e Ct3: classe de tamanho 3.

Figure 1 - Number of individuals per habit and size classes of the lower stratum in 0.5 ha of floodplain forest in APA Ilha do Coumbú, municipality of Belém, Pará.Arv: arboreous, Pal:palm tree, Herb: herbaceous, Arb: arbustive and Lia: liana. Ct1: class size 1, Ct2: class size 2, and Ct3: class size 3. al. (2002), Cattânio et al. (2002), Bianchini et al. (2003), Batista (2008), Brito et al. (2008) e Carim et al. (2008). Quanto à conservação, isso indica a maior intensidade de regeneração do componente arbustivo-arbóreo (KURTZ et al., 2009), o equilíbrio dinâmico da floresta com a constante regeneração (ARAÚJO et al., 2004) e o bom estado de conservação da floresta (BENTESGAMA et al., 2002; BRITO et al., 2008). Para Bianchini et al. (2003), o grande número de indivíduos com baixa estatura estaria relacionado à instabilidade do solo durante períodos de inundação e à pequena profundidade do sistema de raízes.

E. oleracea e $V$. surinamensis foram as mais representativas nos parâmetros fitossociológicos, pelo fato de frutificarem ao longo do ano, pela alta capacidade de germinação, por serem tolerantes à sombra e pela capacidade de suportarem forte estresse hídrico, estabelecendo habilidade mecânica nas raízes para fixação em substratos pouco consolidados em florestas de

Revista Árvore, Viçosa-MG, v.35, n.3, Edição Especial, p.669-677, 2011 


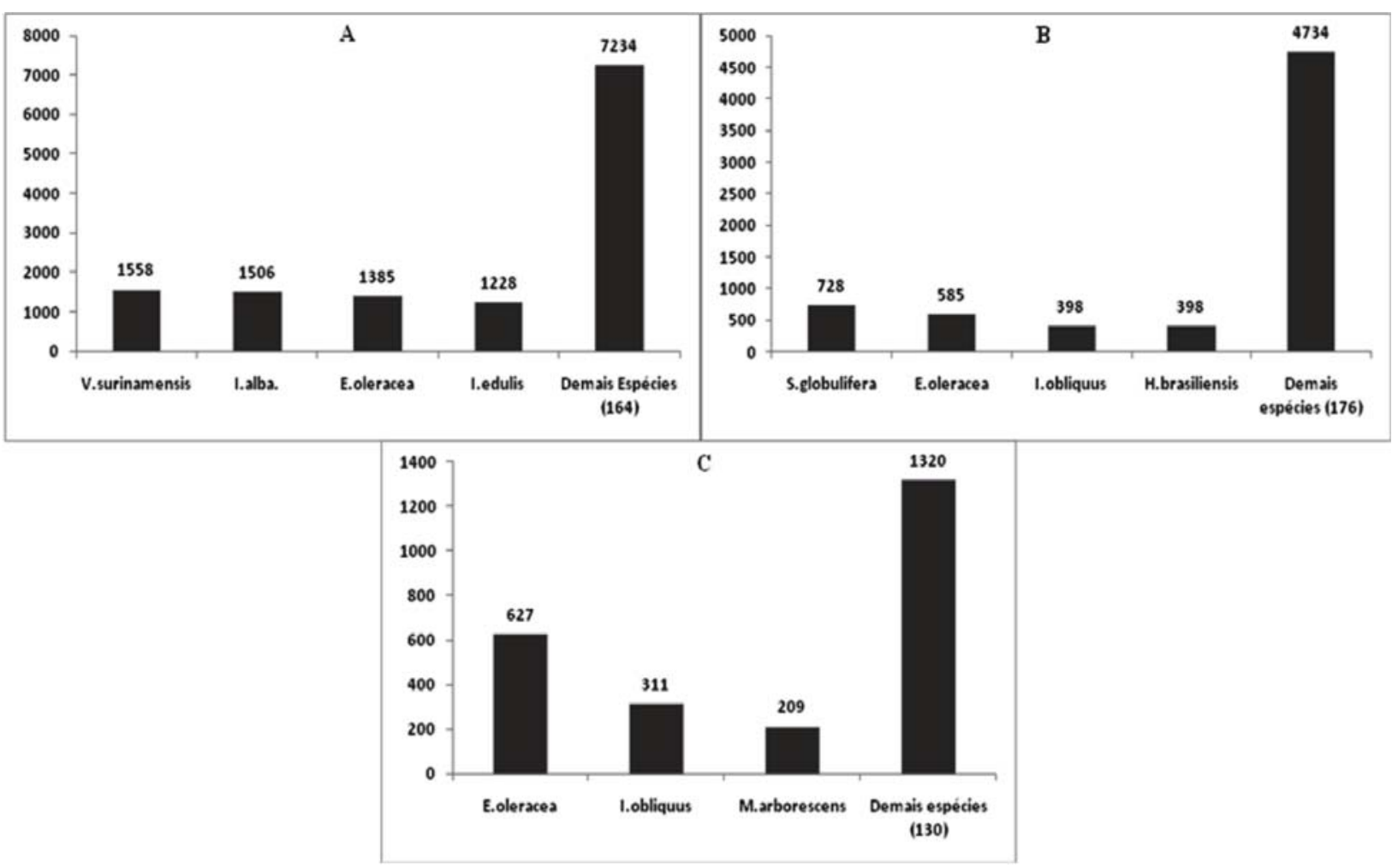

Figura 2 - Número de indivíduos por espécies por classe de tamanho. A - espécies na classe de tamanho 1; B - espécies na classe de tamanho 2; e C - espécies na classe de tamanho 3 do estrato inferior em 0,5 ha de floresta de várzea na APA Ilha do Combu, Município de Belém, Pará.

Figure 2 - Number of individuals per species and size classes. A - species in size class 1; B - species in size class 2 and $C$ - species in size class 3 of the lower stratum in 0.5 ha of floodplain forest from the APA Ilha do Combú, municipality of Belém, Pará.

várzea no Pará (CATTÂNIO et al., 2002; GAMA et al., 2002, 2003; JARDIM; VIEIRA, 2001; ALMEIDA et al., 2004; JARDIM et al., 2004; JARDIM; MOTA, 2007). Segundo Gama et al. (2002), de Euterpe oleracea se retira o palmito de açaí, que é o produto não madeireiro mais comercializado em área de várzea, enquanto a Virola surinamensis tem sua madeira aproveitada por serrarias e indústrias de compensado.

\section{CONCLUSÃO}

A diversidade florística foi alta nas espécies com hábito arbóreo, principalmente Euterpe oleracea e Virola surinamensis, que predominaram no estrato inferior em consequência da produção anual de frutos e germinação das sementes. O maior número de indivíduos arbóreos nas primeiras classes de tamanho é indicativo de que a floresta se encontra em equilíbrio e em bom estado de conservação, em que algumas espécies sofrem adaptações radiculares para fixação de nitrogênio para suportar os constantes alagamentos.

\section{AGRADECIMENTOS}

Ao Conselho Nacional de Pesquisa e Desenvolvimento Tecnológico/CNPq, pelo financiamento do Projeto Padrões de Diversidade Florística, de Regeneração Natural e do Potencial Aromático em Duas Unidades de Conservação do Estado do Pará, como Subsídios ao Plano de Gestão Ambiental. Edital UniversalMCT/CNPq 15/2007 - Processo: 472260/2007-3.

\section{REFERÊNCIAS}

ALMEIDA, S. M.; AMARAL, D. D.; SILVA, A. S. L. Análise florística e estrutura de florestas de várzea no estuário amazônico. Acta

Amazonica, v.34, n.4, p.513-524, 2004. 
THE ANGIOSPERM PHYLOGENY GROUP - APG II. An update of the Angiosperm Phylogeny Group classification for the orders and families of flowering plants: APG II. Botanical Journal of the Linnean Society, v.141, p.399-436. 2003.

ARAujo, M. M. et al. Análise de agrupamento da vegetação de um fragmento de floresta estacional decidual aluvial, cachoeira. Ciência Florestal, v.14, n.1.p.133-147, 2004.

ATWEEL, B. J.; STEER, B. T. The effect of oxygen deficiency on uptake and distribution of nutrients in maize plants. Plant and Soil., v.122, p.1-8, 1990.

BATISTA, F. J. Análise florística e estrutural de florestas de várzea da Resex Chocoré-Mato Grosso, Pará, Brasil. 2008. 88f. Dissertação (Mestrado em Botânica) - Universidade Federal Rural da Amazônia, Belém, 2008.

BENTES-GAMA, M. M.; SCOLFORO, J. R. S.; GAMA, J. R. V. Potencial produtivo de madeira e palmito de uma floresta secundária de várzea baixa no estuário amazônico. Revista Árvore, v.26, n.3, p.311-319, 2002.

BIANCHINI, E. et al. A diversidade e estrutura de espécies arbóreas em área alagável do município de Londrina, Sul do Brasil. Acta Botanica Brasilica, v.17, n.3, p.405-419, 2003.

BRITO, E. R.; MARTINS, S. V.; OLIVEIRA-FILHO, A. T. Estrutura fitossociológica de um fragmento natural de floresta inundável em área de campo sujo, Lagoa da Confusão, Tocantins. Acta Amazonica, v.38, n.3, p.379-386, 2008.

BUDKE, J. C. et al. Floristica e fitossociologia do componente arbóreo de uma floresta ribeirinha, arroio Passo das Tropas, Santa Maria, RS, Brasil. Acta Botanica Brasilica, v.18, n.3, p.581-589, 2004.

CARIM, M. J. V.; JARDIM, M.A.G.; MEDEIROS, T. D. S. Composição floristica e estrutura de floresta de várzea no município de Mazagão, Estado do Amapá, Brasil. Scientiae

Forestalis, v.36, n.79, p.191-201, 2008.
CATTÂNIO, J. H.; ANDERSON, A. B.; CARVALHO, M. S. Floristic composition and topographic variation in a tidal floodplain forest in the Amazon Estuary. Revista Brasileira de Botânica, v.25, n.4, p.419-430, 2002.

FINOL, U. H. Nuevos parámetros a considerarse en el analisis estrutural de las selvas virgens tropicales. Revista Florestal Venezuelana, v.14, n.21, p.337-1144, 1971.

GAMA, J. R. V. et al. Tamanho de parcela e suficiência amostral para estudo da regeneração natural em floresta de várzea na Amazônia. Cerne, v.7, n.2, p.1-11, 2001.

GAMA, J. R. V.; BOTELHO, S. A.; BENTESGAMA, M. M. Composição florística e estrutura da regeneração natural de floresta secundária de várzea baixa no estuário amazônica. Revista Árvore, v.26, n.5, p.559-566, 2002.

GAMA, J. R. V. et al. Estrutura e potencial futuro de utilização da regeneração natural de floresta de várzea alta no município de Afuá, estado do Pará. Ciência Florestal, v.13, n.2, p.71-82, 2003.

JACKSON, M. B.; DREW, M. C. Effects of flooding on growth and metabolism of plant herbaceous. In: KOSZLOWSKI, T. T. (Ed.). Flooding and plant growth. New York: Academic Press, 1984. p.48-128.

JARDIM, M. A. G. Morfologia e ecologia do açaizeiro (Euterpe oleracea Mart.) e das etnovariedades espada e branco em ambiente de várzea do estuário amazônico. 2000. 119f. Tese (Doutorado em Ciências Biológicas) Universidade Federal do Pará, Belém, 2000.

JARDIM, M. A. G.; VIEIRA, I. C. G. Composição florística e estrutura de uma floresta de várzea do estuário Amazônico, Ilha do Combu, estado do Pará. Brasil. Boletim do Museu Paraense Emílio Goeldi, Série Botânica, v.17, n.2, p.333-354, 2001.

JARDIM, M. A. G.; MOTA, C. G. Biologia floral de Virola surinamensis (Rol.) Warb (Myristicaceae). Revista Árvore, v.31, n.6, p.1155-1162, 2007.

KRAMER, K. U.; GREEN, P. S. Pteridophytes and Gymnosperms. In KUBITZKI, K. (Ed.). The families and genera of vascular plants. Berlin, Springer-Verlag, 1990. p.1-404. 
KOZLOWSKI, T. T.; PALLARDY, S. G. Effect of flooding on water, carbohydrate and mineral relations. In: KOZLOWSKI, T. T. (Ed.). Flooding and plant growth. London: Academic Press, 1984. p.165-193.

KURTZ, B. C.; SÁ, C. F. C.; SILVA, D. O. Fitossociologia do componente arbustivoarbóreo de florestas semidecíduas costeiras da região de emerenças, Área de Proteção Ambiental do Pau Brasil, Armação dos Búzios, Rio de Janeiro, Brasil. Rodriguésia, v.60, n.1, p.129146, 2009.

LIMA FILHO, D. A. L. et al. Regeneração natural de três hectares de floresta ombrófila densa de terra firme na região do rio URUCU-AM, BRASIL. Acta Amazonica, v.32, n.4. p.555-569, 2002.

MAGURRAN, A. E. Ecological diversity and its measurement. New Jersey: Princenton University Press, 1988. 179p.

NARVAES, I. S.; LONGHI, S. J.; BRENA, D. A. Florística e classificação da regeneração natural em floresta ombrófila mista na floresta nacional de São Francisco de Paula, RS. Ciência Florestal, v.18, n.2, p.233-245, 2008.

OLIVEIRA, A.N.; AMARAL,I.L. Aspectos florísticos, fitossociológicos e ecológicos de um sub-bosque de terra firme na Amazônia Central, Amazonas, Brasil. Acta Amazonica, v.35, n.1, p.1-16, 2005.
PIELOU, E. C. Mathematical ecology. New York: Wiley, 1977. 165p.

RABELO, F. G. et al. Regeneração natural de florestas esturianas na região do rio AmazonasAmapa-Brasil. Revista de Ciências Agrárias, v.34, p.129-137, 2000.

RIBEIRO et al. Flora da Reserva Ducke: Guia de identificação de plantas vasculares de uma floresta de terra firme na Amazônia Central. Manaus: INPA, 1999. 816p.

RODRIGUES, L. M. B. et al. Composição florística e usos das espécies vegetais de dois ambientes de floresta de várzea. Revista Brasileira de Farmácia, v.87, n.2, p.45-48, 2006.

RICHARDS, P. W. The tropical rain Forest: na ecological study. 2.ed. Cambridge: Cambridge University Press, 1981. 450p.

SANTOS, S. R. M.; MIRANDA, I. S.; TOURINHO, M. M. Análise florística e estrutural de sistemas agroflorestais das várzeas do rio Juba, Cametá, Pará. Acta Amazonica, v.34, n.2, p.251-263, 2004.

SILVA, C. A. R.; SAMPAIO, L. S. Speciation of phosphorus in a tidal floodplain forest in the Amazon estuary. Mangrove Salt Marshes, v.2, n.1, p.51-57, 1998.

SMITH, A. R. et al. A classification of extant ferns. Táxon, v.55, n.3, p.705-731, 2006. 
